\title{
The strange evolution of the Large Magellanic Cloud Cepheid OGLE-LMC-CEP1812
}

\author{
Hilding R. Neilson ${ }^{1}$, Robert G. Izzard ${ }^{2,3}$, Norbert Langer ${ }^{3}$, and Richard Ignace ${ }^{4}$ \\ ${ }^{1}$ Department of Astronomy \& Astrophysics, University of Toronto, 50 St. George Street, Toronto, ON, M5S 3H4, Canada \\ e-mail: neilson@astro.utoronto.ca \\ ${ }^{2}$ Institute of Astronomy, University of Cambridge, Madingley Road, Cambridge, CB3 OHA, UK \\ 3 Argelander-Institut für Astronomie, Universität Bonn, Auf dem Hügel 71, 53121 Bonn, Germany \\ ${ }^{4}$ Department of Physics \& Astronomy, East Tennessee State University, Box 70652, Johnson City, TN 37614, USA
}

Received 10 June 2015 / Accepted 10 August 2015

\section{ABSTRACT}

\begin{abstract}
Classical Cepheids are key probes of both stellar astrophysics and cosmology as standard candles and pulsating variable stars. It is important to understand Cepheids in unprecedented detail in preparation for upcoming Gaia, James Webb Space Telescope (JWST) and extremely-large telescope observations. Cepheid eclipsing binary stars are ideal tools for achieving this goal, however there are currently only three known systems. One of those systems, OGLE-LMC-CEP1812, raises new questions about the evolution of classical Cepheids because of an apparent age discrepancy between the Cepheid and its red giant companion. We show that the Cepheid component is actually the product of a stellar merger of two main sequence stars that has since evolved across the Hertzsprung gap of the HR diagram. This post-merger product appears younger than the companion, hence the apparent age discrepancy is resolved. We discuss this idea and consequences for understanding Cepheid evolution.
\end{abstract}

Key words. binaries: eclipsing - stars: evolution - stars: variables: Cepheids - stars: late-type

\section{Introduction}

Classical Cepheids have been crucial for the understanding of stellar astrophysics and cosmology since the discovery of the Cepheid Leavitt law more than a century ago (Leavitt 1908). They have been used to measure the Hubble constant (Hubble 1929; Riess et al. 2011; Freedman et al. 2012) to a precision of $2 \%$ as well as to constrain the detailed physics of stellar structure and evolution (e.g., Bono et al. 2000), yet there are still a number of mysteries regarding these stars.

One such challenge is the detailed calibration of the Cepheid Leavitt law, i.e., the period-luminosity (PL) relation. In the forthcoming era of the James Webb Space Telescope, it is expected that we will be able to measure the Hubble constant to less than $1 \%$ precision (Freedman \& Madore 2010), but this requires measurements of Cepheid fundamental parameters to unprecedented accuracy along with independent measurements of Cepheid distances. The Gaia satellite is currently operating and will measure distances to thousands of Galactic Cepheids (Windmark et al. 2011), which will complement Large Magellanic Cloud (LMC) Cepheids for which distances are known.

One enduring uncertainty is the decades-old Cepheid mass discrepancy (Cox 1980), in which Cepheid masses measured using stellar evolution and pulsation calculations differ by about 10-20\% (Keller 2008). Bono et al. (2006) suggested four possible resolutions to this discrepancy: changes in the radiative opacities, rotation, convective core overshooting in main-sequence progenitors and stellar mass loss. Neilson et al. (2011) showed that pulsation-driven mass loss can partly explain the mass discrepancy, while Anderson et al. (2014) proposed rotation as an alternative solution. Convective core overshooting has also been shown to resolve the mass discrepancy (Cassisi \& Salaris 2011). The solution to the mass discrepancy might simply be a combination of all three and understanding which physical processes are important will constrain both evolution and pulsation models. However, an ideal method to constrain the Cepheid mass discrepancy is to measure Cepheid masses independently.

Cepheid distances and masses can be measured in eclipsing binary systems. Evans et al. (2005) suggested that about 35\% of all Galactic Cepheids are in spectroscopic binary systems, but none are known to be in eclipsing binary systems. Evans et al. (2013) presented a catalogue of binary companions detected using ultraviolet spectral observations, where the companions are all about $2 M_{\odot}$, inferring a larger binary fraction of $60 \%$. Evans et al. (2015) report radial velocity measurements which further refine the measured binary fraction of Cepheids, noting that what has been measured is a minimum possible binary fraction. Neilson et al. (2015) show that a binary fraction of about $60 \%$ is consistent with the observed binary fraction of main-sequence B-type stars from Kouwenhoven \& de Grijs (2008).

Three eclipsing binary Cepheids have been discovered in the LMC (Soszynski et al. 2008). These eclipsing binary systems provide unique mass and distance estimates that can be compared to estimates using Cepheid evolution and pulsation models. These help to resolve the Cepheid mass discrepancy and constrain the PL relation in greater detail.

Pietrzyński et al. (2010) presented detailed observations of one such eclipsing binary, OGLE-LMC-CEP0227 (CEP0227), with a 310-day orbit and a mass ratio between the components of $q=1.00 \pm 0.01$. One binary component is a classical Cepheid and the companion is a red giant star. The authors measured a Cepheid mass $M=4.14 \pm 0.05 M_{\odot}$ which is consistent with 
stellar pulsation estimates. Cassisi \& Salaris (2011) compared the observed fundamental parameters with stellar evolution calculations and found that evolutionary models agree with measured parameters if one assumes moderate convective core overshooting, a result confirmed by other studies (Neilson \& Langer 2012; Prada Moroni et al. 2012). Pilecki et al. (2013) analyzed the binary light curve in greater detail to measure the projection factor, which is crucial for using the Baade-Wesselink method to measure distances (Baade 1926; Storm et al. 2011a,b; Ngeow et al. 2012; Neilson et al. 2012c). This specific eclipsing binary system constrains both the Cepheid mass discrepancy and the calibration of the distance scale.

Gieren et al. (2014) presented new observations of another eclipsing binary OGLE-LMC-CEP1718, which appears to be composed of two equal mass Cepheids both pulsating in the first overtone. That system has an orbital period of 413 days, but observations are limited and do not independently constrain each star's radius and mass, making conclusions about their evolution difficult. This system appears similar to CEP0227.

While analysis of CEP0227 helps resolve problems, the binary system OGLE-LMC-CEP1812 (CEP1812) appears to create problems. Pietrzyński et al. (2011) measured the masses and radii of the two stars in a 551-day orbit, but found that the Cepheid appears to be about 100 Myr younger than its red giant companion. This result raises questions about the evolution of this binary system. While the binary CEP0227 is consistent with stellar evolution calculations, the binary CEP1812 is not, even though the Cepheid mass is consistent with pulsation calculations. Pietrzyński et al. (2011) hypothesized that the Cepheid captured the red giant companion into a binary orbit at some point during its evolution.

In this article, we hypothesize that the Cepheid star in the system CEP1812 evolved from the merger of two main-sequence stars. We discuss our stellar evolution code and models in Sect. 2 and present measurements of the age difference between the two stars in the systems using those models in Sect. 3. In Sect. 4, we compute stellar evolution models with changing mass consistent with our merger scenario and show that our hypothesis resolves the age discrepancy. In Sect. 5 we discuss the implications of a stellar merger scenario for understanding Cepheid binaries and Cepheid structure and evolution.

\section{Stellar evolution models}

We computed stellar evolution models using the Yoon \& Langer (2005) code. This code has been used to study massive star evolution, supernova progenitors and gamma-ray bursts (e.g. Cantiello et al. 2009; Brott et al. 2011). We also used this code to explore the evolution of classical Cepheids (Neilson et al. 2012a,b; Neilson 2014). Models were computed assuming moderate convective core overshooting, consistent with that measured for the LMC binary Cepheid OGLE-LMC-CEP0227 (Cassisi \& Salaris 2011; Neilson \& Langer 2012; Prada Moroni et al. 2012). In this case, we write the convective core overshooting efficiency as $0.2 H_{\mathrm{P}}$, which is a fraction of the pressure scale height (Brott et al. 2011). Convective core overshooting during main-sequence evolution acts to create a more massive helium core, leading to a more luminous giant star. It also acts to prolong main-sequence evolution and changes the measured ages of post-main-sequence stars. The models included the de Jager et al. (1988) mass-loss prescription for cool stars and the Kudritzki et al. (1989) recipe for hot stars. We did not assume enhanced mass loss during the Cepheid stage of evolution (Neilson et al. 2012a,b). The models assume a composition based on the

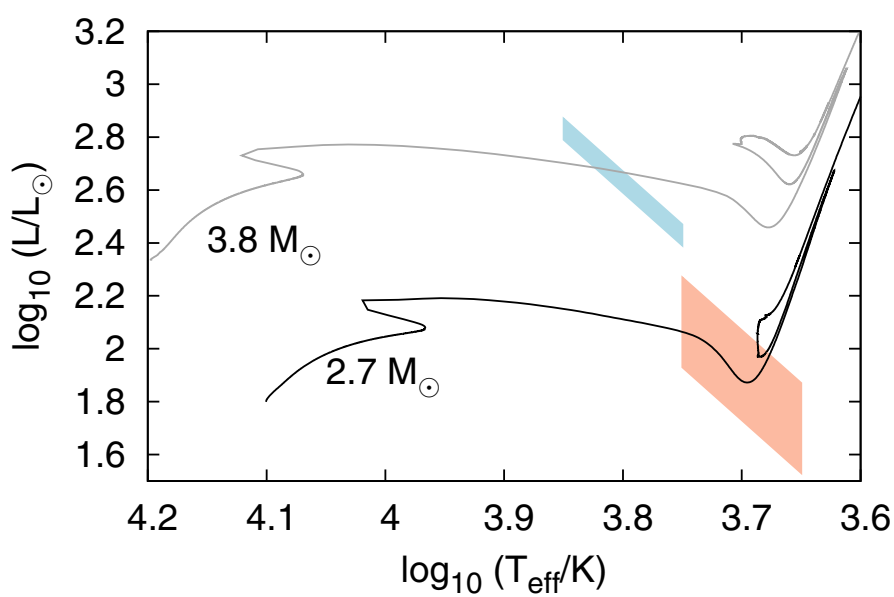

Fig. 1. Hertzsprung-Russell diagram showing stellar evolution models with initial masses $M_{1}=3.8 M_{\odot}$ (gray) and $M_{1}=2.7 M_{\odot}$ (black) along with regions consistent with the radius of the Cepheid, $R_{\text {Cepheid }}=17.4 \pm$ $0.9 R_{\odot}$ (blue) and that of the red giant companion, $R_{\mathrm{RG}}=12.1 \pm 2.3 R_{\odot}$ (red).

Grevesse \& Sauval (1998) solar abundances scaled to the standard LMC metallicity $Z=0.008$ along with an initial helium abundance $Y=0.256$ (Brott et al. 2011).

Stellar evolution models are constrained by the measured mass and radius of each component of OGLE-LMC-CEP1812. The Cepheid has mass $M=3.74 \pm 0.06 M_{\odot}$ and radius $R=$ $17.4 \pm 0.9 R_{\odot}$, while the red giant companion has mass $M_{\mathrm{RG}}=$ $2.64 \pm 0.4 M_{\odot}$ and radius $R_{\mathrm{RG}}=12.1 \pm 2.3 R_{\odot}$. Unfortunately, Pietrzyński et al. (2011) did not measure the effective temperature of either star, so we have fewer constraints for CEP1812 than for CEP0227 (e.g. Cassisi \& Salaris 2011).

Given the masses and radii of the two stars in the binary system, we computed stellar evolution models with initial masses, $M_{1}=3.8$ and $M_{2}=2.7 M_{\odot}$. The stellar evolution tracks are plotted in Fig. 1 along with the regions of the HertzsprungRussell diagram consistent with the measured radii. Stellar evolution models appear to fit the measured stellar masses and radii. However, the models suggest that the Cepheid has an age of about 175 Myr while the red giant star's age is between 420 and 450 Myr. Pietrzyński et al. (2011) noted that the stellar ages are approximately $190 \mathrm{Myr}$ and $369 \mathrm{Myr}$ for the Cepheid and red giant, respectively, based on stellar evolution tracks from Pietrinferni et al. (2004). These model age differences are due to different amounts of convective core overshooting assumed in the models. Including overshooting in stellar evolution models lengthens the main-sequence lifetime and the greater the amount of overshooting the longer the main-sequence lifetime. Regardless of which models are considered, there is a significant age difference. Pietrzyński et al. (2011) suggested the binary system may have formed by stellar capture. We suggest an alternative hypothesis: the apparently younger Cepheid is a stellar merger product between two main-sequence stars in what was originally a triple system that has since evolved across the Hertzsprung gap.

The evolution of the Cepheid in the binary system is also notable because the star appears to be crossing the instability strip for the first time. This phase of evolution is short, about $10^{5}$ years for stars with mass of about $3-4 M_{\odot}$. As such, the number of first-crossing Cepheids relative to the number of Cepheids evolving on the blue loop is small, typically about a few percent (Neilson et al. 2012b). This fact makes CEP1812 more special 

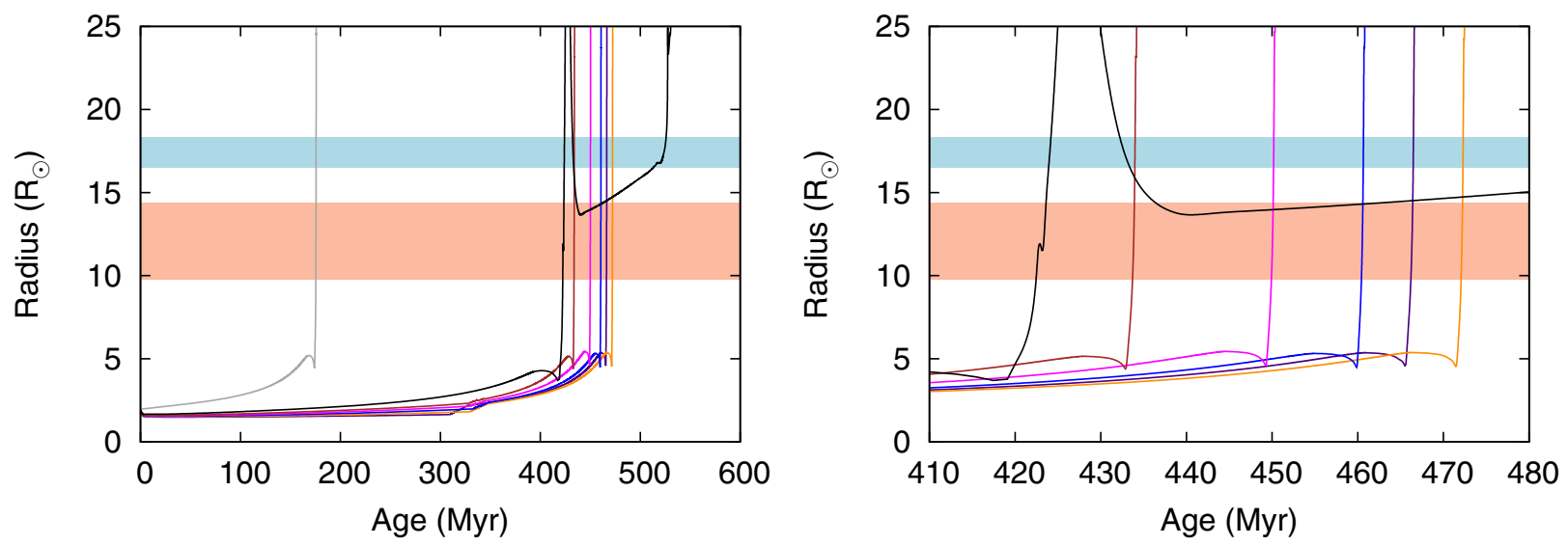

Fig. 2. Left: stellar radii as a function of age for stellar evolution models with initial mass $M_{1}=3.8$ (gray) and $M_{2}=2.7 M_{\odot}$ (black), along with stellar merger models with progenitor initial masses 2.1 (violet), 2.2 (orange), 2.3 (blue), 2.4 (pink) and $2.5 M_{\odot}$ (brown). The blue and red colored regions represent the measured Cepheid and red giant companion radii, respectively. Right: a closer view of the stellar radius as a function age consistent with the current age of the red giant.

as a target for understanding stellar evolution and the transition from the main sequence to the red giant branch.

\section{Merger models}

Stellar mergers appear across the HR diagram, from the formation of blue straggler and sdB/O stars (Mateo et al. 1990; Schneider et al. 2014), many massive stars (de Mink et al. 2013), cool R and J stars (Izzard et al. 2007; Zhang \& Jeffery 2013), R Coronae Borealis stars (Clayton 2013) and anomalous Cepheids (Bono et al. 1997). The coalescence of binary companions can occur via orbital disruptions by third bodies (Kozai 1962; Perets \& Kratter 2012) or through tidal interactions and Roche Lobe overflow (e.g. Hut 1981). Stellar mergers occurring during main-sequence evolution quickly settle and evolve in the same manner as a main-sequence star formed with mass similar to the sum of the merger progenitors (Glebbeek et al. 2013). This merger rejuvenates the star, making it appear younger, which is consistent with the observed age discrepancy between the Cepheid and its red giant companion in CEP1812.

We propose that the Cepheid component of the eclipsing binary system CEP1812 is the result of a merger between two lower mass main-sequence stars that have evolved to become a Cepheid. This progenitor system would have been a hierarchical triple system in which the red giant was originally the most massive star. Because of its eccentric orbit (currently about 0.13 ), the outer star (now the red giant) would have induced Kozai oscillations decreasing the orbital separation between the two lower mass stars until tides and Roche lobe overflow dominate the evolution. The two stars then coalesced rapidly to form a $3.8 M_{\odot}$ star that appears significantly younger than its companion.

This hypothesis is tested by computing a stellar evolution model with mass $M_{1}$ and adding mass $\left(3.8 M_{\odot}-M_{1}\right)$ after about 310-330 million years of evolution. At this age, both stars are evolving on the main sequence. The mass is accreted over a short timescale (relative to the main-sequence lifetime of about $10 \mathrm{Myr}$ ). The main effect of accreting mass is to increase the convective core mass of the star by mixing additional hydrogen, hence, rejuvenating the star. To first order, this mixing is independent of the merging timescale and given the masses we consider, additional mixing effects are not as important (Glebbeek et al. 2013). The merged star then evolves to the end of red giant evolution beyond the first crossing of the Cepheid instability strip where Cepheid is observed to be. The initial model is assumed to have mass greater than the accreted companion, i.e. $M_{1}>3.8 M_{\odot}-M_{1}$, and the donor and accretor have the same surface chemical composition. This is a simplistic calculation that ignores mass lost from the system, which is $\approx 0.1 M_{\odot}$ (Glebbeek $\&$ Pols 2008), and ignores issues related to mixing caused by accretion. The test is sufficient, however, to understand whether a stellar merger resolves the age discrepancy.

We plot the stellar radius as a function of age in Fig. 2. This plot demonstrates the age discrepancy between the $3.8 M_{\odot}$ Cepheid and its $2.7 M_{\odot}$ red giant companion. We also plot stellar merger models with progenitor initial masses $2.1,2.2,2.3,2.4$, and $2.5 M_{\odot}$, which each accrete mass until they reach $3.8 M_{\odot}$ and then evolve normally. Those stellar evolution models are rejuvenated and do not cross the Hertzsprung gap until they are much older than the main-sequence lifetime of stars with that initial mass. The merger models all have a radius consistent with that of the Cepheid at approximately the same age as the red giant star, implying that CEP1812 is the result of a stellar merger that occurred early in the main-sequence evolution of a hierarchical triple system. However, this solution is not unique, other stellar mass combinations are possible depending on when they merge.

\section{Discussion}

There exists a specific class of Cepheids that appear to have evolved from main-sequence stellar mergers: anomalous Cepheids (Bono et al. 1997). They are typically found in older stellar populations in close proximity to RR Lyrae and horizontal branch stars, primarily in dwarf spheroidal satellite galaxies (e.g. Mateo et al. 1995; Kinemuchi et al. 2008). These stars pulsate with periods ranging from about 0.3 to about 2.5 days and LMC anomalous Cepheids have a predicted average mass $M_{\mathrm{AC}}=1.2 \pm 0.2 M_{\odot}$ (Fiorentino \& Monelli 2012). Their masses tend to be smaller than that of LMC Cepheids and there is a small overlap for the longest-period anomalous Cepheid and shortestperiod classical Cepheids. Because of these properties, Sills et al. (2009) suggested they are the merger product between two lowmass main-sequence stars that have evolved across the Cepheid instability strip, akin to the models computed in this work, however Bono et al. (1997) showed that a many ACs are low-mass core helium-burning stars evolving from the horizontal branch. The anomalous Cepheids do not appear to be related to classical Cepheids, but could CEP1812 be a missing link between these two classes of Cepheids? 


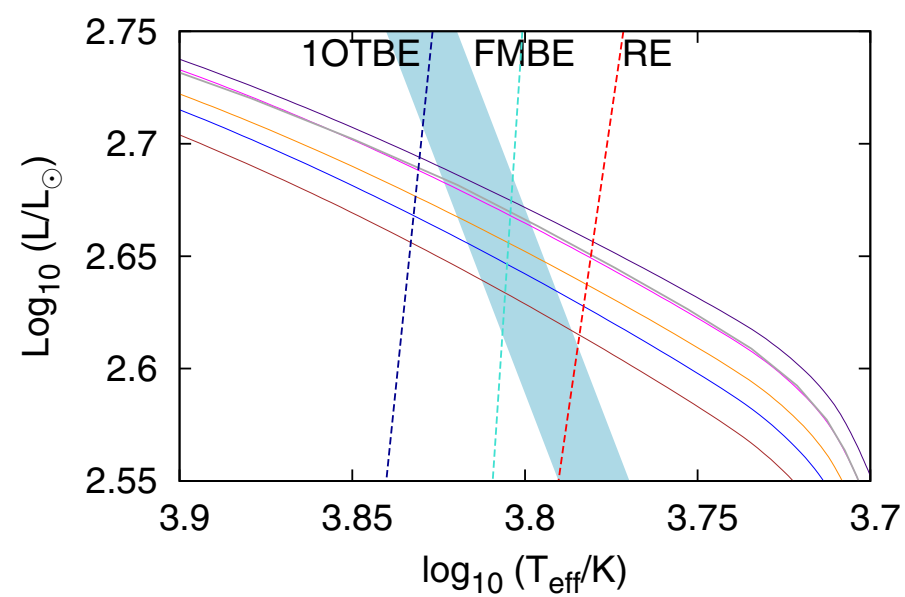

Fig. 3. Hertzsprung-Russell diagram showing stellar evolution models evolving across the Hertzsprung gap and the first crossing of the Cepheid instability strip. Solid lines follow evolution models with initial mass $M_{1}=3.8 M_{\odot}$ (gray), along with stellar merger models with progenitor initial masses 2.1 (violet), 2.2 (orange), 2.3 (blue), 2.4 (pink) and $2.5 M_{\odot}$ (brown). The blue region represents the measured Cepheid radius while the dashed lines denote the blue edge of the first-overtone instability strip "1OTBE" (dark blue), the boundary between first-overtone and fundamental-mode instability strips "FMBE" (light blue) and the red edge of the Cepheid instability strip "RE" (red) (Bono et al. 2000).

We explore this by plotting our stellar evolution models in a Hertzsprung-Russell diagram in Fig. 3. Our merger models evolve across the HR diagram with luminosities consistent with the $3.8 M_{\odot}$ single star model. This implies that CEP1812 is not likely to be a traditional classical Cepheid, but is an anomalous Cepheid (Fiorentino et al. 2006). This possibility is contentious because ACs typically have masses of about one solar mass and have lower metallicities. Because of its mass, CEP1812 likely has a metallicity similar to the assumed LMC metallicity. The star is also not core helium burning. Both properties are inconsistent with various pulsation models of ACs (Bono et al. 1997; Caputo et al. 2004; Marconi et al. 2004). However, a massive AC evolving across the Hertzsprung gap would be rare because this stage of evolution is short, less than $1 \mathrm{Myr}$.

Soszynski et al. (2008) classified CEP1812 as a classical Cepheid based on Fourier decomposition of the observed light curves (Simon \& Lee 1981). Measured Fourier parameters allow for the classification of variable stars because the predicted Fourier parameters tend to cluster for different variable stars and different pulsation modes. However, Fourier parameters for anomalous Cepheids (ACs) vary significantly and the anomalous Cepheid Fourier parameters from the OGLE-III survey of the LMC overlap with those of short-period classical Cepheids including CEP1812 (Fiorentino \& Monelli 2012). The Fourier components for CEP1812 are consistent with both being a shortperiod classical Cepheid or a first-overtone AC. This is somewhat surprising as the Fourier component $\phi_{21}$ only has a small range of values for LMC ACs as measured by Soszyński et al. (2008). This result adds credence to the possibility that CEP1812 is an AC. Pietrzyński et al. (2011) noted that the pulsation period and brightness of CEP1812 is consistent with the measured period-luminosity relation (Soszynski et al. 2008). However, it is also consistent with the first-overtone AC period-luminosity relation (Ripepi et al. 2014). Both its pulsation period and amplitude are consistent with that of other LMC ACs in the OGLE-III survey (Soszyński et al. 2008) again suggesting that
CEP1812 may be an AC and not a classical Cepheid. Based on our models and the fact that CEP1812 is consistent with the pulsation properties of other LMC anomalous Cepheids, we suggest that CEP1812 is not a classical Cepheid but is instead an anomalous Cepheid. Thus, CEP1812 is the most massive AC discovered and is a factor of two more massive than those in the Fiorentino \& Monelli (2012) sample, where the mass is measured from period-luminosity-color relations.

It is not surprising that massive anomalous Cepheids are rare. Stellar mergers are, themselves, rare, but the most noticeable difference between a single star evolving and a stellar merger is that the latter may appear to be younger. Blue straggler stars, which are easy to detect in a globular cluster because the majority of stars there are very old are an example of this. We dectects ACs with the same method: they tend to be found in old populations along with horizontal branch and RR Lyrae stars. It is this contrast that allows anomalous Cepheids to be detected and that also explains why they all tend to be about one solar mass. Only low-mass stars have lifetimes long enough to both undergo a stellar merger and appear in old populations; massive stars that merge would have disappeared long before the general population had significantly evolved. A merger between a 2.4 and a $1.4 M_{\odot}$ star would not appear significantly rejuvenated relative to nearby field stars, but does stand out in a binary system.

Although we suggest that CEP1812 could be classified as an $\mathrm{AC}$, it is not clear that the star will have any properties significantly different from other classical Cepheids. Langer (2012) suggested that strong magnetic fields, typically about $1 \mathrm{kG}$, that are found in about $10 \%$ of intermediate-mass and massive mainsequence stars (Donati \& Landstreet 2009) could be generated in a main-sequence merger. If this hypothesis is correct then CEP1812 could presently have a weak magnetic field that might affect various pulsation properties. Another test of our merger scenario is the abundance of CEP1812. If the star is the product of a merger of two main-sequence stars, where the mass donor has undergone some hydrogen burning then one might expect some chemical anomalies, particularly in the surface helium, nitrogen and carbon abundances. However, because this is a lowmass merger, any changes to the carbon and nitrogen abundances are small, about a fraction of a dex (Glebbeek et al. 2013).

Because the star is a merger product, one might expect some abundance anomalies. However, our model assumes that the merger is a product of two stars with the same initial composition, hence any anomalies would be small. While CEP1812 is a merger of two main-sequence stars, some anomalous Cepheids might be created by the merger between a main-sequence star and an evolved helium-burning star, which would generate a new helium-burning star with an envelope that is massive relative to the total stellar mass, or conversely the core mass is much lower than for a single star at a similar stage of evolution (de Mink et al. 2013). These post-main-sequence mergers could also evolve to become Cepheids but have very different properties because they have a smaller luminosity for a given mass and a more massive envelope leading to different pulsation properties.

In summary, CEP1812 may be an anomalous Cepheid that appears to be just like any classical Cepheid such that it may be the missing link between the two classes of stars. However, the strange evolution of CEP1812 implies that it might be unwise to use this star as a calibrator for the Cepheid Leavitt Law or for resolving the Cepheid mass discrepancy.

Acknowledgements. This work has been supported by a research grant from the NSF (AST-0807664). RGI acknowledges funding from the Alexander von Humboldt Foundation and the Science \& Technology Facilities Council. 


\section{References}

Anderson, R. I., Ekström, S., Georgy, C., et al. 2014, A\&A, 564, A100

Baade, W. 1926, Astron. Nachr., 228, 359

Bono, G., Caputo, F., Santolamazza, P., Cassisi, S., \& Piersimoni, A. 1997, AJ, 113, 2209

Bono, G., Castellani, V., \& Marconi, M. 2000, ApJ, 529, 293

Bono, G., Caputo, F., \& Castellani, V. 2006, Mem. Soc. Astron. It., 77, 207

Brott, I., Evans, C. J., Hunter, I., et al. 2011, A\&A, 530, A116

Cantiello, M., Langer, N., Brott, I., et al. 2009, A\&A, 499, 279

Caputo, F., Castellani, V., Degl'Innocenti, S., Fiorentino, G., \& Marconi, M. 2004, A\&A, 424, 927

Cassisi, S., \& Salaris, M. 2011, ApJ, 728, L43

Clayton, G. C. 2013, in 18th European White Dwarf Workshop., eds. K. J. Ski,

G. Stachowski, P. Moskalik, \& K. Bajan, ASP Conf. Ser., 469, 133

Cox, A. N. 1980, ARA\&A, 18, 15

de Jager, C., Nieuwenhuijzen, H., \& van der Hucht, K. A. 1988, A\&AS, 72, 259

de Mink, S. E., Langer, N., Izzard, R. G., Sana, H., \& de Koter, A. 2013, ApJ, 764,166

Donati, J.-F., \& Landstreet, J. D. 2009, ARA\&A, 47, 333

Evans, N. R., Carpenter, K. G., Robinson, R., Kienzle, F., \& Dekas, A. E. 2005 , AJ, 130, 789

Evans, N. R., Bond, H. E., Schaefer, G. H., et al. 2013, AJ, 146, 93

Evans, N. R., Berdnikov, L., Lauer, J., et al. 2015, AJ, 150, 13

Fiorentino, G., \& Monelli, M. 2012, A\&A, 540, A102

Fiorentino, G., Limongi, M., Caputo, F., \& Marconi, M. 2006, A\&A, 460, 155

Freedman, W. L., \& Madore, B. F. 2010, ARA\&A, 48, 673

Freedman, W. L., Madore, B. F., Scowcroft, V., et al. 2012, ApJ, 758, 24

Gieren, W., Pilecki, B., Pietrzynski, G., et al. 2014, ApJ, 786, 80

Glebbeek, E., \& Pols, O. R. 2008, A\&A, 488, 1017

Glebbeek, E., Gaburov, E., Portegies Zwart, S., \& Pols, O. R. 2013, MNRAS, 434, 3497

Grevesse, N., \& Sauval, A. J. 1998, Space Sci. Rev., 85, 161

Hubble, E. 1929, Proc. National Academy of Science, 15, 168

Hut, P. 1981, A\&A, 99, 126

Izzard, R. G., Jeffery, C. S., \& Lattanzio, J. 2007, A\&A, 470, 661

Keller, S. C. 2008, ApJ, 677, 483

Kinemuchi, K., Harris, H. C., Smith, H. A., et al. 2008, AJ, 136, 1921
Kouwenhoven, M. B. N., \& de Grijs, R. 2008, A\&A, 480, 103

Kozai, Y. 1962, AJ, 67, 591

Kudritzki, R. P., Pauldrach, A., Puls, J., \& Abbott, D. C. 1989, A\&A, 219, 205

Langer, N. 2012, ARA\&A, 50, 107

Leavitt, H. S. 1908, Annals of Harvard College Observatory, 60, 87

Marconi, M., Fiorentino, G., \& Caputo, F. 2004, A\&A, 417, 1101

Mateo, M., Harris, H. C., Nemec, J., \& Olszewski, E. W. 1990, AJ, 100, 469

Mateo, M., Fischer, P., \& Krzeminski, W. 1995, AJ, 110, 2166

Neilson, H. R. 2014, A\&A, 563, A48

Neilson, H. R., \& Langer, N. 2012, A\&A, 537, A26

Neilson, H. R., Cantiello, M., \& Langer, N. 2011, A\&A, 529, L9

Neilson, H. R., Engle, S. G., Guinan, E., et al. 2012a, ApJ, 745, L32

Neilson, H. R., Langer, N., Engle, S. G., Guinan, E., \& Izzard, R. 2012b, ApJ, 760, L18

Neilson, H. R., Nardetto, N., Ngeow, C.-C., Fouqué, P., \& Storm, J. 2012c, A\&A, 541, A134

Neilson, H. R., Schneider, F. R. N., Izzard, R. G., Evans, N. R., \& Langer, N. 2015, A\&A, 574, A2

Ngeow, C.-C., Neilson, H. R., Nardetto, N., \& Marengo, M. 2012, A\&A, 543, A55

Perets, H. B., \& Kratter, K. M. 2012, ApJ, 760, 99

Pietrinferni, A., Cassisi, S., Salaris, M., \& Castelli, F. 2004, ApJ, 612, 168

Pietrzyński, G., Thompson, I. B., Gieren, W., et al. 2010, Nature, 468, 542

Pietrzyński, G., Thompson, I. B., Graczyk, D., et al. 2011, ApJ, 742, L20

Pilecki, B., Graczyk, D., Pietrzyński, G., et al. 2013, MNRAS, 436, 953

Prada Moroni, P. G., Gennaro, M., Bono, G., et al. 2012, ApJ, 749, 108

Riess, A. G., Macri, L., Casertano, S., et al. 2011, ApJ, 730, 119

Ripepi, V., Marconi, M., Moretti, M. I., et al. 2014, MNRAS, 437, 2307

Schneider, F. R. N., Izzard, R. G., de Mink, S. E., et al. 2014, ApJ, 780, 117

Sills, A., Karakas, A., \& Lattanzio, J. 2009, ApJ, 692, 1411

Simon, N. R., \& Lee, A. S. 1981, ApJ, 248, 291

Soszynski, I., Poleski, R., Udalski, A., et al. 2008a, Acta Astron., 58, 163

Soszyński, I., Udalski, A., Szymański, M. K., et al. 2008b, Acta Astron., 58, 293

Storm, J., Gieren, W., Fouqué, P., et al. 2011a, A\&A, 534, A94

Storm, J., Gieren, W., Fouqué, P., et al. 2011b, A\&A, 534, A95

Windmark, F., Lindegren, L., \& Hobbs, D. 2011, A\&A, 530, A76

Yoon, S.-C., \& Langer, N. 2005, A\&A, 443, 643

Zhang, X., \& Jeffery, C. S. 2013, MNRAS, 430, 2113 